\title{
On Uniqueness of KMS States of One-dimensional Quantum Lattice Systems
}

\author{
Huzihiro Araki^ \\ Institut für Theoretische Physik der Universität, Göttingen, Federal Republic of Germany
}

Received May 17, 1975

\begin{abstract}
Uniqueness of KMS states is proved for one-dimensional quantum lattice system. Sakai's theorem on uniqueness of KMS states is generalized to cases of non-commutative generators.
\end{abstract}

\section{§ 1. Introduction}

Uniqueness of equilibrium states for one-dimensional lattice system has been proved by Ruelle [7] for classical interactions and by Araki [1] for quantum interactions with a finite-range interaction. Simpler proofs have since been given for these cases (for example, see [8]. Also see Theorem 2 in [5]). It amounts to showing that any two states $\varphi_{1}$ and $\varphi_{2}$ satisfying the KMS condition are majorized by each other: $\varphi_{1} \leqq \lambda \varphi_{2} \leqq \lambda^{2} \varphi_{1}$ for some $\lambda>0$.

We present here a proof of the uniqueness for one-dimensional quantum lattice system with an interaction $\Phi$, which satisfies the same type of condition as known classical cases, namely surface energy has a bound independent of the volume. The key argument in the proof is Lemma 2 which states roughly that if the relative entropy of a state $\varphi_{1}$ with respect to a state $\varphi_{2}$ is finite, then the associated representation $\pi_{1}$ quasi-contains $\pi_{2}$.

To state the result more precisely, we use the following notation: The $C^{*}$-algebra $\mathfrak{A}$ under investigation will have the following structure as usual: For each integer $v, \mathfrak{A}$ has a subalgebra $\mathfrak{U}_{v}$ mutually commuting for different $v$. For any subset $I$ of the set $Z$ of all integers, $\mathfrak{Y}(I)$ denotes the $C^{*}$-subalgebra of $\mathfrak{A}$ generated by $\mathfrak{A}_{v}, v \in I$. We assume that each $\mathfrak{A}_{v}$ is a type I finite factor and $\mathfrak{A}(Z)=\mathfrak{A}$. For each finite subset $\Lambda$ of $Z$, an interaction potential $\Phi(\Lambda) \in \mathfrak{A}(\Lambda)$ is given such that

(0) $\Phi(\emptyset)=0$,

(1) $\|\Phi\|_{\alpha} \equiv \sup _{v} \sum_{\Lambda}\left\{e^{\alpha N(\Lambda)}\|\Phi(\Lambda)\| ; v \in \Lambda\right\}<\infty$,

where $N(\Lambda)$ denotes the number of points in $\Lambda$ and $\alpha>0$,

(2) the following element $W\left(\Lambda_{n}\right)$ of $\mathfrak{A}$ for an increasing sequence of finite subsets $A_{n}$ of $Z$ is bounded in norm uniformly in $n$ :

$$
W(\Lambda) \equiv \sum_{J}\left\{\Phi(J) ; J \subset C Z, J \cap \Lambda \neq \emptyset, J \cap \Lambda^{\mathrm{c}} \neq \emptyset\right\} .
$$

Here $\Lambda^{\mathrm{c}}$ denotes the complement of $\Lambda$ in $Z$ and $\subset \subset$ denotes a finite subset. Japan.

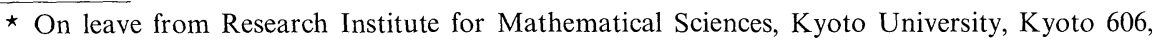


The assumption (0) and (1) are sufficient condition for the existence of the limit

$$
\begin{aligned}
\alpha_{t}(Q) & \equiv \lim _{\Lambda} e^{i t U(\Lambda)} Q e^{-i t U(\Lambda)}, \quad Q \in \mathfrak{I}, \\
U(\Lambda) & \equiv \sum_{J}\{\Phi(J) ; J \subset \Lambda\},
\end{aligned}
$$

which defines a one-parameter group $\alpha_{t}$ of automorphisms of $\mathfrak{A}$.

The assumption (2) is the key condition for the uniqueness of equilibrium states and is essentially the same as the classical cases [7].

Our main result:

Theorem 1. For any $\beta$ real, $\mathfrak{A}$ has one and only one $\alpha_{t}$-KMS state at the inverse temperature $\beta$.

The proof will be given under more abstract setting, which leads to a generalization of Sakai's result [8]: Let $\mathfrak{A}$ be a $C^{*}$-algebra generated by an increasing sequence of $C^{*}$-subalgebras $\mathfrak{U}_{n}$ of $\mathfrak{U}$, which are full matrix algebras. Let $\alpha_{t}$ be a one-parameter group of automorphisms of $\mathfrak{U}$ such that $\alpha_{t}(Q)$ is continuous in $t$ for each $Q \in \mathfrak{U}$. Assume that there exists $h_{n}=h_{n}^{*} \in \mathfrak{U}$ for each $n$ satisfying

$$
\left.(d / d t) \alpha_{t}(Q)\right|_{t=0}=i\left[h_{n}, Q\right]
$$

for all $Q \in \mathfrak{U}_{n}$. Let $\tau$ be the unique tracial state on $\mathfrak{A}$ and $\bar{h}_{n} \in \mathfrak{P}_{n}$ be the conditional expectation of $h_{n}: \tau\left(h_{n} Q\right)=\tau\left(\bar{h}_{n} Q\right), Q \in \mathfrak{U}_{n}$.

An abstract version of Theorem 1 is as follows:

Theorem 2. Assume that

$$
\sup _{n}\left\|h_{n}-\bar{h}_{n}\right\| \equiv \lambda<\infty \text {. }
$$

Then $\mathfrak{U}$ has at most one $\alpha_{t}-K M S$ state for each inverse temperature $\beta$.

Remark 1. If there exists $\hat{h}_{n} \in \mathfrak{U}_{n}$ satisfying

$$
\sup _{n}\left\|h_{n}-\hat{h}_{n}\right\|<\infty
$$

then the condition (1.5) is satisfied: $\bar{h}_{n}-\hat{h}_{n}$ is the conditional expectation of $h_{n}-\hat{h}_{n}$, which implies

$$
\left\|\bar{h}_{n}-\hat{h}_{n}\right\| \leqq\left\|h_{n}-\hat{h}_{n}\right\| \text {. }
$$

Hence

$$
\left\|h_{n}-\bar{h}_{n}\right\| \leqq 2\left\|h_{n}-\hat{h}_{n}\right\| \text {. }
$$

Remark. 2. In the concrete case of Theorem 1, we may set $\mathfrak{A}_{n}=\mathfrak{A}\left(\Lambda_{n}\right)$, $h_{n}=U\left(\Lambda_{n}\right)+W\left(\Lambda_{n}\right), \hat{h}_{n}=U\left(\Lambda_{n}\right)$. Then Theorem 2 and Remark 1 implies the uniqueness part of Theorem 1. The existence is well-known. Thus it is sufficient to prove Theorem 2 .

\section{§ 2. Quasi Containment}

Two representations $\pi_{1}$ and $\pi_{2}$ of a $C^{*}$-algebra $\mathfrak{A}$ is said to be quasiequivalent if kernels of $\pi_{1}$ and $\pi_{2}$ coincide and the mapping $\pi_{1}(Q) \rightarrow \pi_{2}(Q), Q \in \mathfrak{A}$, extends to a $*$-isomorphism of weak closures. In the present case, $\mathfrak{U}$ is simple and $\operatorname{ker} \pi_{1}=$ 
ker $\pi_{2}=0$. If a subrepresentation of $\pi_{1}$ is quasi-equivalent to $\pi_{2}$, then $\pi_{1}$ is said to quasi-contain $\pi_{2}$.

Let $\varphi_{1}$ and $\varphi_{2}$ be states of $\mathfrak{A}$. Let $\mathfrak{S}_{j}, \pi_{j}$ and $\Omega_{j}$ be the space, representation and cyclic vector associated with $\varphi_{j}, j=1,2$.

Lemma 1. If $\pi_{1}$ does not quasi-contain $\pi_{2}$, there exists a sequence of projections $e_{m} \in\left(\bigcup_{n} \mathfrak{A}_{n}\right)$ such that

$$
\begin{aligned}
& \lim _{m} \varphi_{1}\left(e_{m}\right)=0, \\
& \lim _{m} \varphi_{2}\left(e_{m}\right)=a>0 .
\end{aligned}
$$

Proof. Consider the representation $\pi=\pi_{1} \oplus \pi_{2}$ on $\mathfrak{H}=\mathfrak{H}_{1} \oplus \mathfrak{H}_{2}$ with vectors $\Phi_{1}=\Omega_{1} \oplus 0$ and $\Phi_{2}=0 \oplus \Omega_{2}$. Let $\mathfrak{M}=\pi(\mathfrak{Q})^{\prime \prime}, 3$ be the center of $\mathfrak{M}$ and $E_{j}$ be the 3 -support of $\Phi_{j}, j=1,2$. A condition that $\pi_{1}$ quasi-contains $\pi_{2}$ is equivalent to $E_{1} \geqq E_{2}$. Since this condition is not satisfied, there exists a non-zero central projection $E$ such that $E E_{1}=0$ and $E \leqq E_{2}$ Since $\pi\left(\bigcup_{n} \mathfrak{A}_{n}\right)$ is dense in $\mathfrak{M}$, there exists a sequence $a_{m} \in \mathfrak{O}_{n(m)}$ (for some $n(m)$ ) satisfying

$$
\lim _{m} \pi\left(a_{m}\right)=E \text {. }
$$

Let $e_{m}$ be the spectral projection of $a_{m}$ for an interval $[1-\delta, 1+\delta]$ where $\delta \in(0,1)$ is fixed. Then $e_{m} \in \mathfrak{A A}_{n(m)}$ and

$$
\lim _{m} \pi\left(e_{m}\right)=E
$$

by a theorem of Kaplansky [6]. Since $E E_{1}=0, E \Phi_{1}=0$. Since $E \leqq E_{2}$ and $E \neq 0$, $E \Phi_{2} \neq 0$. Hence (2.1) and (2.2) are satisfied with $a=\left\|E \Phi_{2}\right\|^{2}>0$.

\section{§ 3. Relative Entropy}

For two states $\varphi_{1}$ and $\varphi_{2}$ of a matrix algebra, the relative entropy is defined by

$$
S\left(\varphi_{1} / \varphi_{2}\right)=\varphi_{2}\left(\log \varrho_{2}\right)-\varphi_{2}\left(\log \varrho_{1}\right)
$$

where $\varrho_{j}$ is the density matrix for $\varphi_{j}$.

For two faithful states of a von Neumann algebra $\mathfrak{M}$ the definition has been extended with a help of relative modular operators [2], [3]. In particular, for a state $\varphi^{h}$ obtained from a faithful state $\varphi$ by a perturbation $h=h^{*} \in \mathfrak{M}$, we have

$$
\begin{aligned}
& S\left(\varphi^{h} / \varphi\right)=-\varphi(h), \\
& S\left(\varphi / \varphi^{h}\right)=\varphi^{h}(h) .
\end{aligned}
$$

If $N$ is a von Neumann subalgebra of $\mathfrak{M}$ and $\varphi_{j}^{N}$ denotes the restriction of $\varphi_{j}$ to $\mathfrak{N}$, the monotonicity

$$
0 \leqq S\left(\varphi_{1}^{N} / \varphi_{2}^{N}\right) \leqq S\left(\varphi_{1} / \varphi_{2}\right)
$$

has been proved for hyperfinite $\mathfrak{M}$ and $\mathfrak{N}$ [2]. (For finite matrices, non-faithful $\varphi_{j}$ are allowed.) 
If $e \in \mathfrak{M}$ is a projection operator, the inequality (3.4) for $\mathfrak{N}$ generated by $e$ and $(1-e)$ yield

$$
\begin{aligned}
S\left(\varphi_{1} / \varphi_{2}\right) \geqq & \varphi_{2}(e) \log \left\{\varphi_{2}(e) / \varphi_{1}(e)\right\} \\
& +\varphi_{2}(1-e) \log \left\{\varphi_{2}(1-e) / \varphi_{1}(1-e)\right\} .
\end{aligned}
$$

Lemma 2. Let $\varphi_{1}$ and $\varphi_{2}$ be states of $\mathfrak{A}$ and $\varphi_{j}^{n}$ denote the restriction of $\varphi_{j}$ to $\mathfrak{U}_{n}$. If

$$
\sup _{n} S\left(\varphi_{1}^{n} / \varphi_{2}^{n}\right) \equiv \lambda_{1}<\infty,
$$

then $\pi_{1}$ quasi-contains $\pi_{2}$ where $\pi_{j}$ is the cyclic representation of $\mathfrak{U}$ associated with $\varphi_{j}$.

Proof. Assume that $\pi_{1}$ does not quasi-contain $\pi_{2}$. By Lemma 1, there exists a sequence of projections $e_{m} \in \mathfrak{U}_{n(m)}$ such that $\varphi_{1}\left(e_{m}\right) \rightarrow 0$ and $\varphi_{2}\left(e_{m}\right) \rightarrow a>0$. Then

$-\varphi_{2}\left(e_{m}\right) \log \varphi_{1}\left(e_{m}\right) \rightarrow+\infty$,

while

$$
\begin{aligned}
& \varphi_{2}\left(e_{m}\right) \log \varphi_{2}\left(e_{m}\right)+\varphi_{2}\left(1-e_{m}\right) \log \varphi_{2}\left(1-e_{m}\right) \geqq-\log 2, \\
& -\varphi_{2}\left(1-e_{m}\right) \log \varphi_{1}\left(1-e_{m}\right) \geqq 0 .
\end{aligned}
$$

These estimates contradicts with the bound (3.6) when $\varphi_{j}^{n(m)}$ and $e_{m}$ are substituted into $\varphi_{j}$ and $e$ of the inequality (3.5).

\section{§ 4. Gibbs Condition}

Let $\mathfrak{U}_{N}^{\prime}$ denote the commutant of $\mathfrak{U}_{N}$ in $\mathfrak{U}$. Then $\mathfrak{U}=\mathfrak{U}_{N} \otimes \mathfrak{U}_{N}^{\prime}$. Let $\tau_{N}$ and $\tau_{N}^{\prime}$ denote the restriction of the tracial state $\tau$ of $\mathfrak{U}$ to $\mathfrak{U}_{N}$ and $\mathfrak{U}_{N}^{\prime}$. Let

$$
\varphi_{N}^{G}(Q)=\tau_{N}\left(e^{-\beta \bar{h}_{N}} Q\right) / \tau_{N}\left(e^{-\beta \bar{h}_{N}}\right)
$$
at $\beta$ if

Let $W(N) \equiv h_{N}-\bar{h}_{N}$. A state $\varphi$ of $\mathfrak{A}$ is said to satisfy the Gibbs condition

(i) The normal extension $\hat{\varphi}$ of $\varphi$ to the weak closure $\mathfrak{M}=\pi_{\varphi}(\mathfrak{\mathfrak { d }})^{\prime \prime}$ of the associated representation is faithful on $\mathfrak{M}$ and

(ii) for every $N, \varphi^{\beta W(N)}=\varphi_{N}^{G} \otimes \varphi_{N}^{\prime}$ for some linear positive functional $\varphi_{N}^{\prime}$ on $\mathfrak{U}_{N}^{\prime}$.

Theorem 3. If $\varphi$ satisfies the KMS condition at $\beta$, it satisfies the Gibbs condition at $\beta$.

Proof. The condition (i) is known to follow from the KMS condition. Let

$$
\psi=\left\{\varphi^{\beta W(N)}(1)\right\}^{-1} \varphi^{\beta W(N)}
$$

be a state on $\mathfrak{A}$ obtained from $\varphi$ by a perturbation $\beta W(N)-\left\{\log \varphi^{\beta W(N)}(1)\right\} 1$.

Let $\sigma_{t}^{\varphi}$ and $\sigma_{t}^{\psi}$ be modular automorphisms of $\mathfrak{M}$ for states $\hat{\varphi}$ and $\hat{\psi}$ (the normal extensions of $\varphi$ and $\psi$ to $\mathfrak{M})$. Then

$$
(d / d t)\left\{\sigma_{t}^{\psi}(x)-\sigma_{t}^{\varphi}(x)\right\}_{t=0}=i \beta\left[\pi_{\varphi}(W(N)), x\right]
$$

for $x \in \mathfrak{M}$. The KMS condition implies

$$
\sigma_{t}^{\varphi}\left(\pi_{\varphi}(Q)\right)=\pi_{\varphi}\left(\alpha_{-\beta t}(Q)\right), \quad Q \in \mathfrak{H} .
$$


By (1.4), (4.4) and (4.3), we obtain

$$
\left.(d / d t) \sigma_{t}^{\psi}\left(\pi_{\varphi}(Q)\right)\right|_{t=0}=-i \beta \pi_{\varphi}\left(\left[\bar{h}_{N}, Q\right]\right)
$$

for $Q \in \mathfrak{A}_{N}$. By the group property,

$$
(d / d t) \sigma_{t}^{\psi}(x)=\sigma_{t}^{\psi}\left\{\left.(d / d s) \sigma_{s}^{\psi}(x)\right|_{s=0}\right\} .
$$

Let

$$
\alpha_{t}^{N}(Q)=e^{i t \bar{h}_{N}} Q e^{-i t \bar{h}_{N}} .
$$

Then

$$
(d / d t) \alpha_{t}^{N}(Q)=i\left[\bar{h}_{N}, \alpha_{t}^{N}(Q)\right] .
$$

From (4.5), (4.6) and (4.8), we obtain

$$
(d / d t) \sigma_{t}^{\psi}\left(\pi_{\varphi}\left\{\alpha_{\beta t}^{N}(Q)\right\}\right)=0
$$

for $Q \in \mathfrak{A}_{N}$. This implies

$$
\sigma_{t}^{\psi}\left\{\pi_{\varphi}(Q)\right\}=\pi_{\varphi}\left\{\alpha_{-\beta t}^{N}(Q)\right\}, \quad Q \in \mathfrak{A}_{N} .
$$

In particular

$$
\pi_{\varphi}\left(\bar{h}_{N}\right) \in \mathfrak{M}^{\psi} .
$$

where the centralizer $\mathfrak{M}^{\psi}$ is the set of $x \in \mathfrak{M}$ invariant under $\sigma_{t}^{\psi}$. If we set $\psi_{1}=\psi^{\beta \bar{h}_{N}}$, then (4.11) implies

$$
\psi_{1}(Q)=\psi\left(e^{\beta \bar{h}_{N}} Q\right), \quad Q \in \mathfrak{A},
$$

and

$$
\sigma_{t}^{\psi_{1}}(Q)=e^{i \beta \pi_{\varphi}\left(\bar{h}_{N}\right)} \sigma_{t}^{\psi}(x) e^{-i \beta \pi_{\varphi}\left(\bar{h}_{N}\right)}
$$

for $x \in \mathfrak{M}$. The last equation together with (4.10) imply

$$
\begin{aligned}
& \pi_{\varphi}\left(\mathfrak{H}_{N}\right) \in \mathfrak{M}^{\psi_{1}} \\
& \text { If } Q_{1}, Q_{2} \in \mathfrak{A}_{N} \text { and } Q^{\prime} \in \mathfrak{A}_{N}^{\prime}, \text { then } \\
& \begin{aligned}
\psi_{1}\left(Q_{1}\left(Q_{2} Q^{\prime}\right)\right) & =\psi_{1}\left(\left(Q_{2} Q^{\prime}\right) Q_{1}\right) \quad(\text { by }(4.14)) \\
& =\psi_{1}\left(Q_{2} Q_{1} Q^{\prime}\right)
\end{aligned}
\end{aligned}
$$

which implies

$$
\psi_{1}\left(\left[Q_{1}, Q_{2}\right] Q^{\prime}\right)=0 .
$$

Since $\mathfrak{A}_{N}$ is a full matrix algebra, any element $Q \in \mathfrak{A}_{N}$ can be written as

$$
Q=\tau_{N}(Q) 1+\sum_{j}\left[Q_{j 1}, Q_{j 2}\right]
$$

for some $Q_{j 1}, Q_{j 2} \in \mathfrak{A}_{N}$. Hence (4.15) implies

$$
\psi_{1}\left(Q Q^{\prime}\right)=\tau_{N}(Q) \psi_{1}\left(Q^{\prime}\right)
$$

for $Q \in \mathfrak{U}_{N}, Q^{\prime} \in \mathfrak{A}_{N}^{\prime}$. Namely $\psi_{1}=\tau_{N} \otimes \psi_{1}^{\prime}$ where $\psi_{1}^{\prime}$ is the restriction of $\psi_{1}$ to $\mathfrak{Q}_{N}^{\prime}$. Because of (4.12), we obtain (ii) of the Gibbs condition. 
Remark. What we need in the subsequent application is a part of the Gibbs condition, which says that the restriction of $\varphi^{\beta W(N)}$ to $\mathfrak{I}_{N}$ is the Gibbs state $\varphi_{N}^{G}$ up to a normalization constant $\varphi^{\beta W(N)}(1)$. This much is deduced immediately from (4.10) by the uniqueness of KMS states for full matrix algebra.

\section{§ 5. Proof of Theorem 2}

Let $\varphi_{\infty}$ be any one of the accumulation points of the sequence of states $\varphi_{n}^{G} \otimes \tau_{n}^{\prime}$ at $n=\infty$. Let $\varphi$ be an arbitrary extremal $\alpha_{t}$-KMS state at $\beta$. By a known general result, $\varphi$ is primary.

Let $p$ be a fixed positive integer. Since $\mathfrak{A}_{p}$ is of finite dimension and $\varphi_{\infty}$ is a weak accumulation point of $\varphi_{n}^{G} \otimes \tau_{n}^{\prime}$, there exists an integer $N(\varepsilon)$ for any given $\varepsilon>0$ such that $N(\varepsilon) \geqq p$ and

$$
\left\|\left(\varphi_{N(\varepsilon)}^{G}\right)_{p}-\left(\varphi_{\infty}\right)_{p}\right\|<\varepsilon
$$

where $(\varphi)_{p}$ denotes the restriction of $\varphi$ to $\mathfrak{A}_{p}$. Note that $\left(\varphi_{N}^{G}\right)_{p}=\left(\varphi_{N}^{G} \otimes \tau_{N}^{\prime}\right)_{p}$ for $N \geqq p$.

By (3.4), we have

$$
0 \leqq S\left((\varphi)_{p} /\left(\psi_{N}\right)_{p}\right) \leqq S\left(\varphi / \psi_{N}\right)
$$

where $\psi_{N}$ denotes the state $\psi$ given by (4.2). By (3.3), we have the following estimate:

$$
\begin{aligned}
S\left(\varphi / \psi_{N}\right) & =\psi_{N}(\beta W(N))-\log \varphi^{\beta W(N)}(1) \\
& \leqq \psi_{N}(\beta W(N))-\varphi(\beta W(N)) \\
& \leqq 2|\beta| \lambda
\end{aligned}
$$

where we have used (1.5) and the following Peierls-Bogolubov inequality [4]

$$
\log \varphi^{\beta W(N)}(1) \geqq \varphi(\beta W(N))
$$

which follows from $S\left(\psi_{N} / \varphi\right) \geqq 0$ for example.

By the Gibbs condition, $\left(\psi_{N}\right)_{p}=\left(\varphi_{N}^{G}\right)_{p}$ for $N \geqq p$. Hence (5.2) and (5.3) imply

$$
0 \leqq S\left((\varphi)_{p} /\left(\varphi_{N}^{G}\right)_{p}\right) \leqq 2|\beta| \lambda \text {. }
$$

The function $\operatorname{tr}(\varrho \log \varrho)$ of the density matrices $\varrho$ for a finite dimensional case is bounded and continuous. If $\sigma$ is strictly positive, $\operatorname{tr}(\varrho \log \sigma)$ is also bounded and continuous as a function of $\varrho$. Hence

$$
S\left((\varphi)_{p} /\left(\varphi_{\infty}\right)_{p}\right)=\lim _{\varepsilon \rightarrow 0} S\left((\varphi)_{p} /\left(\varphi_{N(\varepsilon)}^{G}\right)_{p}\right)
$$

due to (5.1). By (5.4), we obtain

$$
0 \leqq S\left((\varphi)_{p} /\left(\varphi_{\infty}\right)_{p}\right) \leqq 2|\beta| \lambda
$$

Since $p$ is any positive integer, Lemma 2 implies that the cyclic representation $\pi$ associated with $\varphi$ quasi-contains the cyclic representation $\pi_{\infty}$ associated with $\varphi_{\infty}$. Since $\pi$ is primary, this implies that $\pi$ and $\pi_{\infty}$ are quasiequivalent. Since $\varphi_{\infty}$ is fixed, any primary KMS states are mutually quasiequivalent. The proof of Theorem 2 is then completed by the following Lemma. 
Lemma 3. If two extremal $K M S$-states $\varphi$ and $\varphi^{\prime}$ of a $C^{*}$-algebra $\mathfrak{A}$ at the same $\beta$ have quasi-equivalent associated cyclic representations, then $\varphi=\varphi^{\prime}$.

Proof. Let $\mathfrak{H}, \pi$ and $\Omega$ be canonically associated with $\varphi$ and $\mathfrak{M}=\pi(\mathfrak{U})^{\prime \prime}$. Since $\varphi$ is a KMS-state, $\Omega$ is separating (and cyclic by definition). By quasi-equivalence, there exists $\Omega^{\prime} \in V_{\Omega}^{1 / 4}$ such that the associated vector states is $\varphi^{\prime}$, where $V_{\Omega}^{1 / 4}$ denotes the natural positive cone (see [3], for example). Since $\varphi^{\prime}$ is a KMSstate, $\Omega^{\prime}$ is separating for $\mathfrak{M}$ and hence is also cyclic (see [3], for example). Let the unitary cocycle (the intertwining operator for modular automorphisms) be denoted by

$$
u_{t}^{\varphi \varphi^{\prime}}=\Delta_{\Omega^{\prime}, \Omega}^{i t} \Delta_{\Omega}^{-i t} .
$$

Since the KMS condition characterizes the modular automorphisms, we have $\sigma_{t}^{\varphi^{\prime}}=\sigma_{t}^{\varphi}\left(=\pi_{\varphi} \alpha_{-\beta t} \pi_{\varphi}^{-1}\right.$ on $\left.\pi_{\varphi}(\mathfrak{U})\right)$ and hence

$$
u_{t}^{\varphi \varphi^{\prime}} \in \mathfrak{M} \cap \mathfrak{M}^{\prime} .
$$

Since $\varphi$ is an extremal KMS state, the center $\mathfrak{M} \cap \mathfrak{M}^{\prime}$ is trivial and hence $u_{t}^{\varphi \varphi^{\prime}}=e^{i c t}$ for some real $c$. By analytic continuation, we have

$$
\Omega^{\prime}=\mathcal{u}_{-i / 2}^{\varphi^{\prime} \varphi} \Omega=e^{c / 2} \Omega .
$$

Hence $\varphi=\varphi^{\prime}$.

Acknowledgement. The present work is completed while the author is a Gauß Professor of the Göttingen Academy of Sciences. The author would like to thank the Akademie der Wissenschaften zu Göttingen for financial support. Hospitality of Professor H. J. Borchers and members of the Institut für Theoretische Physik, Universität Göttingen, is gratefully acknowledged.

\section{References}

1. Araki, H.: Commun. math. Phys. 14, 120-157 (1968)

2. Araki, H.: Commun. math. Phys. 38, 1-10 (1974)

3. Araki,H.: Recent developments in the theory of operator algebras and their significance in theoretical physics. To appear in Proceedings of convegno sulle algebre $C^{*}$ e loro applicazioni in Fisica Teorica, Rome, 1975

4. Araki, H.: Commun. math. Phys. 34, 167-178 (1973)

5. Dobrushin, R. L.: Funktsional'nyi Analiz i Ego Prilozheniya 2 (4), 44-57 (1968)

6. Kaplansky,I.: Pacific J. Math. 1, 227-232 (1951)

7. Ruelle, D.: Commun. math. Phys. 9, 267-278 (1968)

8. Sakai, S.: On commutative normal *-derivations II (preprint)

H. Araki

Research Institute

for Mathematical Sciences

Kyoto University

Kyoto 606, Japan 
